\title{
«GOOD TRAINING FOR GOOD JUDGMENTS». EL PROGRAMA HELP (HUMAN RIGHTS EDUCATION FOR LEGAL PROFESSIONALS) DE FORMACIÓN EN DERECHOS HUMANOS DEL CONSEJO DE EUROPA
}

\author{
RAFAEL BUSTOS GISBERT \\ Catedrático $(A)$ de Derecho Constitucional \\ Profesor Escuela Judicial/Focal Point HELP \\ EVA PASTRANA \\ Directora de HELP
}

SUMARIO

I. El marco normativo de la formación en derechos humanos en el Consejo de Europa. II. HELP: una red de formación de profesionales jurídicos. III. HELP: una metodología específica. El papel de una Tutora HELP. IV. Una oferta de cursos de formación en estándares europeos de derechos humanos. V. Conclusión.

\section{EL MARCO NORMATIVO DE LA FORMACIÓN EN DERECHOS HUMANOS EN EL CONSEJO DE EUROPA}

El 12 de mayo de 2004 el Comité de Ministros del Consejo de Europa aprueba su Recomendación (2004)4 sobre la Convención Europea de Derechos Humanos en la educación universitaria y en la formación profesional. Previamente diversos textos habían abordado, parcialmente, la cuestión relativa a la formación en derechos humanos en Europa. Entre estos instrumentos de soft Law del Consejo de Europa destacan la Resolución (78)41 sobre la enseñanza de Derechos Humanos; la Resolución (78)40 sobre investigación en derechos humanos y la Recomendación (85) 70 sobre enseñanza de los derechos humanos en las escuelas.

En la Rec. (2004)4 se apunta ya la importancia que el Consejo de Europa va a conferir a la formación en derechos humanos por dos motivos esenciales que se van a reiterar en todos los documentos posteriores. En primer lugar, la conexión existente entre formación en derechos humanos y el correcto funcionamiento del principio de subsidiariedad. En virtud de este principio, los órganos de garantía de los derechos previstos en el Convenio Europeo de Derechos Humanos $(\mathrm{CEDH})$ no entran en funcionamiento hasta que 
no se hayan agotado las instancias internas de protección de los derechos. Es pues a las autoridades nacionales, y muy en particular a las judiciales, a las que corresponde en primer lugar la aplicación y salvaguardia de los derechos de las y los ciudadanos europeos ${ }^{1}$. De este modo, la adecuada formación de profesionales en derechos humanos permitiría que estos fueran directamente aplicados en el nivel estatal y no existiera la necesidad de protegerlos en los niveles internacionales.

Con ello se avanzaba en el segundo motivo apuntado en aquel documento: la eficacia preventiva de una buena formación en derechos humanos. Si los derechos son suficientemente conocidos y aplicados en el nivel nacional, la reparación de los mismos se producirá en dicho ámbito territorial y con ello se asegurará su pronta reparación.

Ya en el primer documento en el que seriamente se aborda la cuestión de la formación en materia de derechos humanos subyace un gran principio inspirador de todo el esquema de formación derivado de la actuación del Consejo de Europa. La idea de que un buen funcionamiento de un sistema de derechos se asienta en su correcta defensa y aplicación por parte del juez de primera instancia. De la primera jueza que conoce un caso que suscite una cuestión de derechos. Es este juez quien ha de tutelar correctamente los derechos. Todo lo que venga después de su actuación será una tutela tardía en la que no se habrá evitado la violación del derecho por lo que solo podremos aspirar a su reconocimiento y, en la medida de los posible, a su reparación. Es por ello que el destinatario de la formación en derechos posiblemente no haya de ser la jueza de una instancia última de decisión nacional, sino más bien las profesionales que componen los estadios inferiores en la aplicación del Derecho.

Por ello se recalcaba la importancia tanto de la formación universitaria, como profesional en derechos humanos, especialmente en algunos sectores relativos a la aplicación de la ley y a la administración de justicia. En tal sentido una de las recomendaciones del texto se centra en la garantía de la formación universitaria y profesional en materia de derechos humanos de modo que se incorpore como elemento central en cualquier plan de estudios jurídicos y que se convierta en un componente de los programas para el acceso a las distintas profesiones jurídicas, así como a la formación continua prevista para jueces, fiscales y abogadas. De manera complementaria, y veremos más adelante la importancia de este elemento, se apoyan expresamente las iniciativas dirigidas a la formación de profesoras especializadas y formadores en materia de derechos humanos. De este modo debería garantizarse que los profesores de formadores y las propias formadoras en derechos humanos tuvieran el nivel de conocimiento correspondiente a la evolución de la jurisprudencia del Tribunal Europeo de Derechos Humanos (TEDH) y que además, pudieran satisfacer las necesidades específicas de cada sector profesional objeto de la formación.

Respecto a la primera de las líneas de acción se destacaba la importancia de que la formación universitaria no se hiciera solo desde la perspectiva de los propios derechos en sí mismos, sino que se incorporara como materia horizontal a todas las disciplinas jurídicas. Igualmente se insistía en la promoción de formación de postgrado en materia de derechos humanos.

1 Para evitar el cansancio para el lector del uso del doble género a lo largo del texto, hemos optado por usar indistintamente ambos géneros en el resto del ensayo. 
Resultan mucho más interesantes para estas páginas las consideraciones realizadas en el apéndice de la recomendación respecto a la formación profesional. El punto de partida es claro: un mejor conocimiento de la Convención ayudará a su aplicación en el ámbito interno y con ello se evitarán tanto las violaciones del $\mathrm{CEDH}$, como el planteamiento de demandas manifiestamente infundadas por no cumplir los requisitos de admisibilidad establecidos en el case law del TEDH. Por ello, continúa el texto, una formación específica en los estándares de la CEDH debería incorporarse a los programas no solo de las Facultades de Derecho, sino también en los centros de formación de jueces y fiscales. Con respecto a las abogadas, esta formación debería ser suministrada por los colegios de abogados. Se llegaba incluso a recomendar que estas acciones formativas adoptaran una forma de seminarios de dos días.

Sin embargo, todo el documento subraya un elemento básico: es a las autoridades nacionales a quien corresponde establecer sus programas de formación de acuerdo con sus propias situaciones nacionales.

Este punto de partida respecto a la formación en derechos humanos fomentada desde el Consejo de Europa experimentará un cambio importante a partir de la Declaración de Brighton en abril de 2012 que es donde podemos situar un gran impulso para el «re»-nacimiento del programa HELP.

Debe notarse que la declaración de Brighton se emite en el marco de una conferencia de alto nivel en plena crisis del funcionamiento del TEDH como consecuencia de la carga de trabajo del mismo derivada del crecimiento exponencial de las demandas contra los Estados por violación de los derechos reconocidos en el CEDH. Es pues en el marco de las reformas que acabarán originando la aprobación y puesta en marcha de los Protocolos 14 y 15, así como de las reformas en el funcionamiento del Tribunal respecto a la admisión de demandas en el que hay que situar el impulso a la formación en derechos en el marco del Consejo de Europa.

En el texto de la declaración vamos a encontrar un tercer elemento básico que se va a unir a las previas ideas de subsidiariedad y carácter preventivo de la formación en el marco del sistema del Convenio. Este tercer elemento ya estaba incluido en la declaración de Interlaken (19 de febrero de 2010) pero ahora se va a conectar con la formación. Nos referimos a la idea de que el cumplimiento de los estándares exigidos por el CEDH en la protección de derechos ha de ser una responsabilidad compartida por el Consejo de Europa y por los Estados miembros.

En tal sentido la lucha contra el crecimiento de la carga de trabajo del TEDH (derivado de las violaciones de derechos pero también de la interposición de demandas manifiestamente infundadas) que es el objetivo esencial de la Conferencia va a incluir una petición expresa a que los Estados adoptaran una medida esencial (apartado vi del párrafo 9.a): proveer la apropiada información y formación sobre el CEDH en el estudio inicial, formación continua y desarrollo profesional de juezas, abogados y fiscales. Este sin duda es el pistoletazo que propulsa el programa HELP.

A partir de tal mandato, en el bienio 2013-2014, se intensificará pues en programa específico del Consejo de Europa dirigido a la creación de un mecanismo capaz de ayudar a los Estados en su labor de formación respecto a los estándares europeos en derechos humanos.

De este modo no es sorprendente que en la Declaración de Bruselas (27 de marzo de 2015) la conferencia pida a los Estados que «mejoren la formación de los jueces, fiscales, abogadas y funcionarias en la Convención y su implementación, incluyendo lo relativo a 
la ejecución de sentencias, mediante el aseguramiento de que ello constituye una parte integral de su formación inicial y continua, cuando sea relevante, incluyendo el acceso al programa de Formación en Derechos Humanos para Profesionales Jurídicos (HELP) del Consejo de Europa, así como los programas de formación del Tribunal y sus publicaciones» (Apdo. B1.b del Plan de Acción aprobado en dicha conferencia)

Con todo ello se ha pretendido dar origen a una suerte de círculo virtuoso en el que se pretende reflejar la complementariedad de los instrumentos de defensa de los derechos en el marco del Consejo de Europa establecidos en sus órganos y textos de, por una parte, fijación de estándares y, por la otra, de vigilancia de su implementación. Complementariedad a la que hay de dotar del suficiente impacto en la realidad de la práctica diaria de los Estados. Impacto que solo se puede obtener a través de la cooperación derivada de la formación en los mismos de los profesionales jurídicos.

\section{HELP: UNA RED DE FORMACIÓN DE PROFESIONALES JURÍDICOS}

Básicamente el programa HELP² es una red de formación especializada en derechos humanos y dirigida a profesionales del Derecho. La red está coordinada por el secretariado de HELP encuadrado como una unidad de la Dirección General de Derechos Humanos y Estado de Derecho del Consejo de Europa. La red, además, está formada por las instituciones nacionales de formación de jueces y/o fiscales así como las de abogadas de los 47 Estados miembros del Consejo. En este sentido HELP es una red paneuropea de escuelas de formación profesional en materias vinculadas al Derecho. Para su buen funcionamiento cada institución nacional de formación nombra una persona de contacto permanente: «info points» para las instituciones de formación de abogados y «focal point» para las de formación de juezas y fiscales. Estas personas constituyen el enlace permanente con la red a la vez que el coordinador de las actividades a nivel nacional. Estos puntos de contacto son, sin duda, la clave del buen (o mal) funcionamiento de la red. Son ellas quienes han de recoger las necesidades de formación, contribuir a elaborar proyectos de acciones formativas, ejecutarlos a nivel nacional o transnacional y, en fin, evaluar su eficacia en la consecución de los objetivos.

En esta red se integran como «partners» otras organizaciones con intereses en la formación jurídica profesional en Europa como pueden ser la Red Europea de Escuelas Judiciales (EJTN) o el Consejo Europeo de Colegios de Abogados (CCBE) ${ }^{3}$.

Como tal red persigue los siguientes objetivos (aplicables a petición de las instituciones nacionales):

- apoyar el desarrollo y promoción de la formación inicial y continua de los profesionales jurídicos en los estándares europeos en materia de derechos humanos tal y como son interpretados por el TEDH; help/home

$2 \mathrm{Al}$ programa y a sus recursos se puede acceder a través de su dirección web en : https://www.coe.int/en/web/

3 Otros partners son la Asociación Internacional de Jueces; la Federación Europea de Colegios de Abogados (FBE), asociaciones nacionales de Jueces y Fiscales; Asociaciones profesionales de Abogados; redes de asistencia legal y centros especializados en derechos Humanos, el propio TEDH y la fundación Mémoire Albert Cohen. 
- compartir las mejores prácticas así como proveer ayuda en la elaboración de planes de estudio, materiales de formación y metodologías docentes para los profesionales jurídicos. En tal sentido corresponde a HELP identificar los temas prioritarios para el desarrollo de nuevos cursos o modulos de formación HELP;

- brindar asesoramiento sobre cómo ayudar a los Estados miembros a mejorar la capacitación de juezas, fiscales y abogadas para aplicar el CEDH a nivel nacional mediante la permanente formación;

- suministrar apoyo para mejorar la capacidad de las abogadas para satisfacer los criterios de admisibilidad de las demandas planteadas ante el TEDH aumentando la calidad de las demandas;

- Proporcionar un amplio conocimiento y uso de los recursos de formación de HELP;

- Promover la coordinación de las iniciativas de formación en derechos humanos dirigidas a los profesionales jurídicos y propuestas por instituciones internacionales y nacionales.

HELP celebra en el mes de junio una conferencia anual en Estrasburgo. En la conferencia participan: el secretariado de HELP en el CoE; los representantes de las instituciones nacionales de formación; los focal e info points de los Estados; los partners del programa y todo un conjunto de invitadas especialistas en formación de profesionales jurídicos.

La conferencia es un foro de encuentro de la red en un triple sentido. En primer lugar, es un foro de rendición de cuentas. Durante la misma se exponen las actividades realizadas en el marco de la red y los datos de participación en las acciones formativas diseñadas o apoyadas por HELP. En segundo lugar, es un foro de discusión sobre la propia red. Así, se aprovecha para trabajar en aspectos concretos del funcionamiento y objetivos de la red de manera que se puedan establecer sus grandes líneas de actuación. La conferencia de 2015 trabajó y optó porque la formación en HELP fuera necesariamente interprofesional; la conferencia de 2016 se centró en la aprobación de la metodología de formación específica de la red; la conferencia de 2017 vinculó la red a la formación en derechos humanos con grupos vulnerables, en particular la infancia y en la formación en derechos en los niveles educativos más primarios y, finalmente, la conferencia de 2018 se centró en las relaciones entre la red y los centros de formación superior y en particular las universidades. En tercer y último lugar, la conferencia es un foro de futuro. Durante su celebración se presentan los nuevos cursos que se van a lanzar el año siguiente; se discute en grupos de trabajo el contenido de dichos cursos y se proponen las actividades del año siguiente. En todas las conferencias se elabora un roadmap a partir de las encuestas rellenadas por las participantes (adoptando modos informáticos de interacción) y de las conclusiones de los diferentes grupos de trabajo. El roadmap no solo guiará la actuación de la red durante el siguiente año, sino que será el documento que servirá como base para su valoración durante la conferencia del siguiente año.

La conferencia anual es sin duda uno de los puntos fuertes de la red pues permite a sus miembros encontrarse y establecer relaciones directas y personales que hacen posible los acuerdos previos para desarrollar diferentes actividades de formación en cooperación a lo largo de los siguientes años. De este modo se crea una red informal de contactos y 
confianza entre instituciones de formación de diferentes Estados, niveles y especialidades profesionales que resulta de enorme utilidad para poder realizar formación en derechos humanos novedosa y más completa.

El trabajo de los distintos órganos de la red se ve completado con el desarrollado por un Consultative Board. Como su propio nombre indica es un órgano consultivo formado por seis miembros elegidos por los representantes de las instituciones nacionales de formación de jueces, fiscales y de abogadas durante las conferencias anuales con un mandato de dos años y una sola reelección.

El consejo consultivo evalúa los materiales formativos elaborados por el programa; propone nuevos temas en los que desarrollar actividades formativas; elabora y coordina las estrategias de cooperación con otras unidades de formación; provee asesoramiento en las metodologías; coopera con el personal técnico en la elaboración de los cursos etc. Al final de su mandato, el consejo consultivo presenta un informe ante la conferencia sobre sus actividades y sobre las del programa.

La red además presenta una estructura orientada a la consecución de objetivos de formación sobre base territorial o sobre base material. En el primer sentido se encuentran los proyectos $H E L P$ in the $E U$ (que, financiado por la UE, sustituye al anterior proyecto HELP in the 28 y dirigido a la formación en los Estados miembros de la Unión Europea y su problemática concreta respecto a la relación entre el CEDH y la Carta de Derechos Fundamentales de la Unión Europea); HELP in Russia y HELP in the Western Balkans (específicamente encaminados a la mejora de la formación en estas zonas debido a los problemas de respeto a los estándares europeos que se están produciendo en concreto en esos Estados). Otros proyectos vinculados a la formación en materias específicas son los relativos a la prevención de la radicalización en Europa y a la protección de niños migrantes y refugiados.

\section{HELP: UNA METODOLOGÍA ESPECÍFICA. EL PAPEL DE UNA TUTORA HELP}

La red HELP elaboró una metodología específica aplicada a la formación en los estándares europeos en derechos humanos. Tal metodología se encuentra recogida en las más de 140 páginas del HELP Guidebook on Human Rights Education for legal professionals ${ }^{4}$.

La metodología diseñada pretende adaptarse totalmente a tres tipos de necesidades diferentes. Por una parte, las derivadas de la formación de profesionales jurídicos (juezas, fiscales y abogados) con lo que ello supone de limitaciones de tiempo y de necesidad de estar orientada a la aplicación práctica y no a la reflexión teórica general. Por la otra, la metodología ha de adaptarse a los diferentes ordenamientos jurídicos nacionales. Es cierto que los estándares europeos son comunes. Pero la forma en que éstos actúan y son aplicados varía de un Estado a otro. Este ha de ser un elemento básico a tener en cuenta en el diseño y ejecución de acciones de formación en derechos. Finalmente, la necesidad de que los cursos sean realizados fundamentalmente on line y

$4 \mathrm{El}$ acceso a la guía puede obtenerse directamente en https://rm.coe.int/CoERMPublicCommonSearchServices/DisplayDCTMContent?documentId=090000168066db63 
con el objetivo de convertirse en fórmulas de autoaprendizaje puro, de oferta libre y gratuita.

Un curso es elaborado siguiendo un procedimiento específico y perfectamente establecido previamente desde la identificación del tema de acuerdo con los socios estatales hasta su puesta a disposición al público en general. De este modo se encuentra perfectamente formalizado todo el proceso de creación de un curso de este tipo; desde la primera identificación de una necesidad de formación, hasta la evaluación de su eficacia formativa.

En el marco de este modelo de metodología ocupa un lugar destacado el tutor o formador de los cursos HELP. Esta tutora va a desarrollar las siguientes funciones esenciales en el marco de la metodología elegida:

- Adaptar un curso-modelo HELP a las necesidades de formación del Estado;

- Utilizar los materiales, herramientas y recursos de HELP para elaborar la adaptación del curso al ordenamiento jurídico nacional de que se trate;

- Tutorizar las primeras ediciones del curso que adoptan un formato semipresencial;

- Evaluar las virtudes y defectos de los cursos en su aplicación práctica;

- Proponer los cambios pertinentes tanto en el curso-modelo como en su adaptación;

- Colaborar en la elaboración de otros cursos modelos y en la formación de formadoras HELP.

Los tutores HELP reciben una formación específica suministrada por la red mediante cursos de «Formación de formadoras» (ToT, training of trainers, en su acrónimo inglés). Los aspirantes son seleccionados por los miembros de la red (normalmente a propuesta de las instituciones nacionales de formación) a partir de su formación específica y entre los diferentes tipos de profesionales a quienes va dirigida la formación. En los ToT las tutoras adquieren todos los conocimientos y habilidades necesarios para el diseño y ejecución de un curso HELP. Ello incluye:

i) técnicas de aprendizaje profesional concretas de tipo presencial y semipresencial;

ii) definición de sistemas de determinación de objetivos de formación;

iii) uso de herramientas tecnológicas para la creación de recursos formativos on line;

iv) diseño de instrumentos de interacción en cursos semipresenciales y on line;

v) determinación de sistemas de evaluación del cumplimiento de objetivos de formación;

vi) fórmulas de informe para la mejora de los cursos.

Esta formación es de naturaleza tanto teórica como práctica y adopta una forma semipresencial. Incluye un trabajo de dos días de tipo presencial y el diseño, on line, de cursos aplicando la metodología HELP. Los participantes en el curso son evaluados y aquellos que superan el mismo se convierten en Formadores Certificados del Programa HELP que serán los únicos que pueden tutorizar los cursos ofrecidos en el marco de la plataforma. 


\section{UNA OFERTA DE CURSOS DE FORMACIÓN EN ESTÁNDARES EUROPEOS DE DERECHOS HUMANOS}

Como ya ha quedado expuesto, el programa HELP tiene como objetivo la creación de cursos de formación en derechos humanos dirigidos a los profesionales jurídicos europeos. Para ello se crea la red y se adopta una determinada metodología.

La elaboración y ejecución de los cursos sigue la siguiente estructura.

En primer lugar, una vez identificada las necesidades formativas y los objetivos que se pretenden conseguir con un determinado curso; la secretaría HELP procede a organizar, de acuerdo con la red en general, los contenidos del Curso-Modelo. Este es un paquete SCORM que contiene básicamente la formación en los estándares europeos vigentes en el tema objeto del curso, con especial atención al case Law del TEDH. No se trata sólo de información, sino que se trata de un curso interactivo que incluye actividades y tareas a desarrollar por todo participante. El curso así diseñado está pensado para la formación exclusivamente on line. El Curso-Modelo se elabora en inglés y todas las herramientas (texto on line, sentencias, videos, ejercicios, etc) serán, en principio, en ese idioma.

Elaborado y evaluado por los expertos el Curso-Modelo, se acuerda con las instituciones de formación de los Estados miembros, su lanzamiento en el marco de la oferta formativa de cada uno de ellos. Esto requerirá una colaboración de todo tipo (incluida financiera) entre la secretaría de HELP y los Estados miembros para proceder a la adaptación del Curso-Modelo a cada Estado. En este punto es singularmente importante la colaboración horizontal en el seno de cada Estado entre las instancias de formación nacional de juezas, fiscales y abogados. Una buena cooperación entre ellas asegura que acuerden previamente qué cursos tienen más interés para su Estado y compartan los costes derivados de la adaptación generando sinergias que solo pueden ser positivas 5 .

La adaptación requiere la traducción de los materiales al idioma del país en el que se vaya a impartir y, sobre todo, el diseño y elaboración de los contenidos necesarios para su adaptación al ordenamiento jurídico estatal afectado. Esto es, el Curso-Modelo contiene los estándares europeos en una determinada materia. Pero corresponderá a las instituciones nacionales (a través de un tutor HELP) adaptar esos estándares a su aplicación en un concreto ordenamiento jurídico. De este modo, junto a los contenidos propios del Curso-Modelo europeo se incluirán y desarrollarán los propios del Curso-Adaptado nacionalmente. El curso modelo así como el curso nacionalmente adaptado son colgados en la página web de HELP si bien en esta primera fase son de acceso restringido pues requieren su lanzamiento de común acuerdo por HELP y la institución nacional de formación.

5 El programa HELP ha considerado una buena práctica a seguir por el resto de miembros de la red la cooperación estrechísima entre la Escuela Judicial, el Consejo General de la Abogacía y el Centro de Estudios Judiciales. Esta cooperación ha provocado que todos los cursos lanzados en España estén abiertos a la participación de cualquiera de sus profesionales o que habitualmente los tutores de los cursos sean los mismos con independencia de su especialización (carrera judicial, fiscal o abogacía) y de la institución en concreto que los lanza. 
Antes del lanzamiento del curso la institución nacional de formación procede a la selección de las participantes de acuerdo con sus propios criterios. Idealmente, y esta es la práctica en España, los cursos se abren a las tres profesiones jurídicas afectadas (Juezas, Fiscales y Abogadas) de modo que en los mismos se produzca siempre una participación interdisciplinar, pues este es uno de los rasgos identificadores de la metodología HELP del que derivan indudables ventajas formativas y sinergias de enorme relevancia en el proceso de aprendizaje. Los cursos HELP pretenden ser, además de un instrumento de formación, un foro de diálogo donde las profesionales puedan comprender mejor los diferentes roles que les corresponden en la defensa y aplicación de los estándares europeos de derechos.

El comienzo de la formación se desarrolla en una sesión presencial de lanzamiento donde además de ponencias de contenido, se aborda el uso de las herramientas tecnológicas para el desarrollo on line del resto del curso. En tal sentido, la plataforma de HELP para los cursos es una versión de Moodle. El tutor procede a matricular en el curso a todas las participantes seleccionadas que, tras darse de alta en la citada plataforma HELP, podrán acceder a los contenidos.

El resto del curso se desarrolla enteramente on line. Normalmente, los participantes deberán desarrollar primero los contenidos «europeos», incluidos en el Curso-Modelo y, posteriormente, los módulos «nacionales», incluidos en el Curso-Adaptado. La duración estimada total del Curso-Adaptado no debe ser superior a 30 ó 40 horas (de las cuales de 10 a 20 horas lo serán del Curso-Modelo) de dedicación y en un periodo no superior a 3 meses. Durante el curso el tutor deberá establecer las tareas, regular el acceso a los materiales, moderar los debates, evaluar o supervisar la autoevaluación de los participantes, etc. Finalizado el curso los participantes que lo superen reciben un certificado tanto de la institución nacional de formación como del Consejo de Europa para atestiguar el que se ha superado la acción formativa de que se trate. Igualmente la tutora deberá emitir un informe del curso que acompañe a la evaluación realizada por los participantes.

El curso puede ser lanzado con este formato tantas veces como sea necesario. Normalmente se lanza, al menos, dos veces para comprobar que sus contenidos y herramientas cumplen los objetivos perseguidos y que se alcanzan los resultados de formación esperados. Pero si existe una alta demanda de formación para el tema concreto, se puede lanzar en más ocasiones.

Una vez el curso ha sido comprobado, la versión adaptada es colgada en la plataforma de formación HELP convertida ahora en un curso de formación enteramente on line. Esto es, en un curso de autoformación puro. Para ello se eliminan las herramientas que pudieran reflejar las opiniones de los participantes (como por ejemplo los foros) y se incluye en la oferta general de cursos HELP. Estos cursos son de oferta libre y enteramente gratuita. Cualquier profesional puede matricularse en ellos y adquirir la formación ofrecida.

Finalmente, también las instituciones de formación nacional pueden incorporar el Curso Adaptado a sus propias plataformas de formación en el Estado gestionando directamente su impartición a nivel nacional sin que sea necesaria la participación de HELP. Así, cursos ya probados pueden pasar a formar parte del plan de estudios de la formación inicial o continua de cualquiera de las instituciones de formación de juezas, fiscales o abogados. En el caso español, por ejemplo, el curso de Introducción al CEDH se incluye 
como docencia obligatoria en el plan de estudios de formación inicial de la Escuela Judicial a partir de 2018.

De este modo en la plataforma de formación HELP encontraremos una oferta general de cursos que adaptan una doble estructura. Cursos tutorizados y cursos no tutorizados. Los cursos no tutorizados incluyen distintas versiones adaptadas a algunos de los estados miembros (en función del interés mostrado por los mismos) y cada una en su propio idioma. El Curso-Modelo (representando una duración entre 10-20 horas) aparecerá no solo incluido en tales versiones, sino también separadamente en idioma inglés.

El listado de Cursos-Modelo existente elaborado según los parámetros expuestos es el siguiente (si el Curso-Adaptado está disponible en español se añade entre paréntesis):

1) Admissibility criteria in applications submitted to the ECtHR (Criterios de admisibilidad de una demanda ante el TEDH),

2) Alternative measures to detention,

3) Anti-discrimination,

4) Asylum and the ECHR (Asilo y CEDH; elaborado junto con el ACNUR),

5) Key human rights principles in biomedicine,

6) Business and Human Rights,

7) Child-friendly Justice,

8) Combatting Trafficking in Human Beings,

9) Counterfeiting of Medical Products (Lucha contra la falsificación de medicinas y productos médicos),

10) Data Protection and Privacy Rights (Protección de datos y derecho a la vida privada),

11) Family Law,

12) Fight against Racism, Xenophobia, Transphobia (Lucha contra el racismo, la xenophobia, homophobia y transfobia),

13) Freedom of Expression,

14) Hate crime and Hate speech,

15) Introduction to the ECHR and ECtHR (Introducción al CEDH y al TEDH),

16) International Cooperation in Criminal Matters,

17) Internally Displaced Persons,

18) Labour Rights as Human Rights (Derechos laborales),

19) Pre-trial Investigation in light of the ECHR,

20) Prohibition of Ill-treatment,

21) Property Rights,

22) Refugee and Migrant Children,

23) Reasoning of (Criminal) Judgments,

24) Rights of Persons with Disabilities,

25) Right to Liberty and Security (Art 5),

26) Right to Respect for Private and Family Life (Art ECHR 8),

27) Right to the Integrity of the Person (Bioethics),

28) Transitional Justice,

29) Violence Against Women. 
Actualmente en preparación se encuentran los siguientes cursos:

1) Procedural rights in criminal proceedings and victims' rights,

2) Radicalisation Prevention,

3) Family Law,

4) Human Rights in Sports,

5) Fight against Drugs,

6) CPT standards,

7) Women's Access to Justice.

La evolución de HELP y su progresivo éxito puede comprobarse con los siguientes datos de usuarios 2011 (1534), 2012 (1879), 2013 (3541), 2014 (5191), 2015 (6615), 2016 (9462), 2017 (18071), 2018 (24800). Junto a ello, señalar que los Estados con mayor participación serían Francia (7465), Rusia (1756), Ucrania (1328), España (1252), Italia (994), Turquía (924), Rumanía (849), Reino Unido (519), Albania (500), Azerbaijan (491), Grecia (448), Bosnia Herzegovina (429), Serbia (421), Moldavia (4169 y Lituania (405).

\section{CONCLUSIÓN}

El programa HELP constituye un intento de facilitar el cumplimiento de la responsabilidad compartida que supone asegurar la vigencia de los estándares en materia de derechos humanos en Europa. Late tras el mismo la profunda convicción de que corresponde a las juezas y tribunales nacionales construir la primera e inmediata barrera contra cualquier lesión de tales estándares. Sin la aplicación de tales estándares desde la primera instancia judicial, no extraeremos toda la potencialidad de los criterios establecidos en la rica jurisprudencia de Estrasburgo y no será tan útil la supervisión internacional del comportamiento de los Estados.

Pero implementar estos criterios requiere el manejo de una información y unas herramientas que exigen un conocimiento profundo y permanente de tales estándares. «Good Training for Good Judgements». Es el lema de HELP y describe bien lo que este programa pretende conseguir. Una buena formación para una buena aplicación judicial de los estándares europeos de derechos humanos. Esta formación es la que se propone ofrecer el programa HELP del Consejo de Europa a partir de una estructura reticular, flexible y basada en la cooperación y no en la competencia entre instancias de formación.

Esta es una idea con la que debe acabarse una exposición como la presente. HELP no es una red para sustituir a las instancias de formación de profesionales jurídicos en los Estados miembros. Es una red para colaborar con ellas en el aseguramiento de que los estándares europeos impregnen de abajo hacia arriba toda la actuación judicial de los Estados miembros. Actuación que no puede entenderse solo desde los jueces y magistradas. Sino desde todas las intervinientes en cualquier proceso. Por supuesto, los jueces, pero también los fiscales y las abogadas. Entre todas ellas, con la puesta a disposición de instrumentos sencillos de formación adaptados a sus necesidades, se puede, quizás, alcanzar un objetivo esencial para una auténtica Europa de los Derechos: que los instrumentos y estándares para su protección estén a disposición de cualquier ciudadana en cualquier momento y en cualquier lugar de Europa. 
TITLE: Good training for good judgment: Coucil of Europe's help programme: Human Rights Education for legal professionals

ABSTRACT: This essay exposes the essential features of the HELP program (Council of Europe). HELP (Human Rights Education for Legal Professionals) aims to train European legal standards on buman rights to legal professionals in order to ensure its compliance at the national level, avoiding the resort to European bodies. The program is simultaneously a network, a methodology and an offer of training courses. The final objective is to ensure that through good training it may be possible to reach good (respectful) judgments in human rights.

RESUMEN: El presente trabajo pretende exponer los aspectos esenciales conformadores del programa HELP del Consejo de Europa. Programa que pretende la formación en los estándares europeos en derechos humanos de los profesionales jurídicos con la finalidad de asegurar su cumplimiento en el nivel nacional sin necesidad de acudir a las instancias europeas. El programa es simultáneamente una red, una metodología y una oferta de formación dirigido a asegurar que a través de una buena formación (good training) se consigan unas buenas decisiones judiciales (good judgements) en materia de derechos bumanos.

KEY WORDS: Human Rights Education for Legal Professionals. Council of Europe

PaLABRAS CLAVE: HELP. Consejo de Europa

FECHA DE RECEPCIÓN: 11.07.2018 FECHA DE ACEPTACIÓN: 13.09.2018 